\title{
JamSketch: Improvisation Support System with GA-based Melody Creation from User's Drawing
}

\author{
Tetsuro Kitahara ${ }^{1}$, Sergio Giraldo ${ }^{2}$, and Rafael Ramírez ${ }^{2 \star}$ \\ 1 College of Humanities and Sciences, Nihon University, Tokyo, Japan \\ kitahara@chs.nihon-u.ac.jp \\ 2 Music Technology Group, Universitat Pompeu Fabra, Barcelona, Spain \\ \{sergio.giraldo, rafael.ramirez\}@upf.edu
}

\begin{abstract}
In this paper, we propose a system that enables nonmusicians to enjoy improvisation just by drawing a melodic outline on the piano-roll display. Once the user draws a melodic outline, the system immediately generates a melody using a genetic algorithm, in which the fitness function is calculated based on the similarity to the outline, an N-gram probability, and entropy. Experimental results show that generated melodies have quality similar to performances by non-expert human performers.
\end{abstract}

Keywords: Improvisation, melodic outline, melody generation, algorithmic composition, genetic algorithm

\section{Introduction}

Improvisation is one of the most enjoyable forms of music performance in which musicians create music compositions in real time combining communication of emotions, instrumental technique and spontaneous response to others. Hence, improvisation requires advanced skills and knowledge of music. Many people cannot improvise even if they have skills in playing an instrument. If computing technologies could enable these people to improvise without prior musical knowledge or skill, then they could enjoy music from a broader range of forms.

Thus, there have been several attempts to support non-musicians' improvisation. Parson [14] developed a system that composes improvisational melodies based on a chess game by the user. During a chess game, the user's behaviors in the game are mapped into a musical structure, and are further transmitted to sound generation software. Amiot et al. [1] applied the Fourier transform to musical structures for computer-aided improvisation. Buchholz et al. [2] developed a collaborative improvisation support system called coJIVE, in which the width of each virtual key (a target to hit with the baton) varies according to

\footnotetext{
* This work was supported by JSPS KAKENHI Grant Numbers 26240025, 26280089, 16K16180, 16H01744, 16KT0136, and 17H00749 as well as by the Spanish TIMuL Project (TIN2013-48152-C2-2-R), and the TELMI Project of the Horizon 2020 Research and Innovation Programme (grant agreement No. 688269).
} 


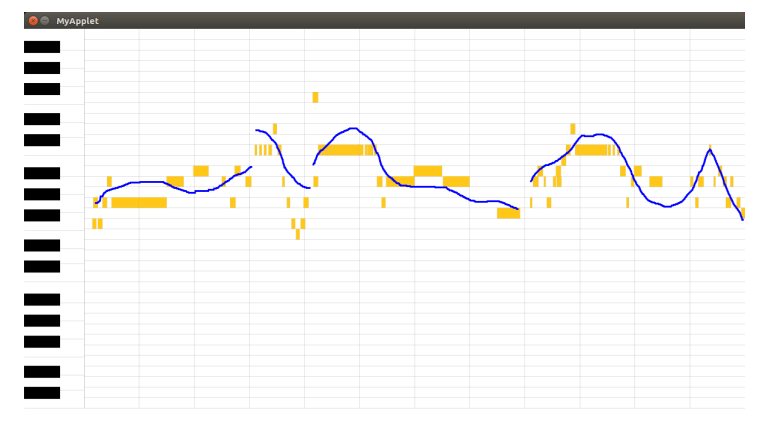

Fig. 1. An example of melodic outlines and screenshots of our system

its musical availability. Miyashita et al. [11] proposed a keyboard that presents the musical availability of each key by controlling its temeperature; i.e., keys for dissonant notes are automatically hot. Ishida et al. [8] developed a system that identifies the musical appropriateness of each note in the user's improvisational performance using the $N$-gram model. If not appropriate, then it automatically corrects it to a musically appropriate note in real time. Fels et al. [5] developed an improvisation support system called RhyMe based on the fixed-function mapping, which is a key-to-note mapping method to maintain the harmonic function of each key. Pachet [13] proposed a system called Continuator that automatically generates stylistically consistent improvisations based on a Markov model. Keller [9] developed music notation software called Impro-visor, in which the user can compose jazz solo melodies using advices from the software.

One important issue in designing an improvisation support system is the design of the human interface for input of musical ideas. The human interface should be easy, intuitive, quickly inputable, and musically meaningful, but also should not require musical knowledge. Most systems described above $[2,11,8$, 5] are based on a traditional keyboard instrument, and they may be difficult for people who cannot play a keyboard instrument. Impro-visor [9] is based on traditional music notation software, so it may be difficult for people who are not familiar with the traditional music notation. The use of a chess game instead of playing an instrument [14] is a novel idea, but it is not easy to design reasonable mapping between situations in the game and musical semantics. If this mapping is not rationally designed, then the user cannot express his/her musical ideas as situations in the game.

In this paper, we propose an improvisation support system using a melodic outline that the user draws (Figure 1). Use of drawing as the user's input is common in music systems for non-musicians. Hyperscore is a graphical music composition tool, in which different colors are assigned to different motives and the user can compose by drawing lines with various colors [4]. Hand Composer recognizes a gesture by the user using Leap Motion and creates music based on the gesture [10]. InkSplorer is a tool for composing contemporary music based on 
hand-written gestures [6]. Music Sketcher is also a drawing-based user interface for electroacoustic music [15]. UPIC, developed by Xenakis, is also an music composition tool based on drawing, in which the user draws on the built-in board and the system interprets the drawing as dynamic envelopes, scores in the timepitch domain, or other musical parameters [17]. In addition, we also developed a melody editing system, in which the outline of a target melody is represented as a curve and the user can edit the melody by redrawing the curve [16]. However, these systems do not focus on the support of improvisation. On the other hand, our system, called JamSketch, is capable of creating melodies in real time based on melodic outlines drawn by the user. Once the user draws outlines on the pianoroll display with the mouse or touch screen, the system immediately generates melodies that are along to the user's outline and musically appropriate using a genetic algorithm. With this melody generation feature, users can experience improvisational music performance.

\section{Issues}

Our goal is to develop a system in which non-musicians can enjoy improvisation with a simple operation. As discussed in Introduction, we have two issues:

\section{Issue 1: Human interface}

We assume that our users have neither musical knowledge nor skills in playing an instrument. The system must allow users to input their musical ideas in an intuitive and easy way. Next, users should input them during musical accompaniment because our aim is to support improvisation, not off-line composition. The human interface should therefore be quickly inputable. Third, users have to realize a reasonable mapping between operators in the human interface and musical semantics in order to express their musical ideas with this interface. Of course, the human interface should not require users to have music knowledge.

We adopt melodic outlines as users' input data. Melodic outlines are a subsymbolic representation of melodies in which note-level information (the onsets, durations, and pitch of each note) is hidden. An example of melodic outlines is shown in Figure 1. By drawing a melodic outline, the user can specify the overall contour of the melody to be performed.

Melodic outlines satisfy all of these requirements. First, melodic outlines are easy and intuitive to input. Second, melodic outlines can be input quickly; it typically takes a few seconds to input a 4-bar melodic outline. Third, melodic outlines have a rational mapping to melodic semantics. Also, no expert knowledge of music is required to draw melodic outlines.

\section{Issue 2: Melody generation}

Various techniques have been proposed to generate melodies [12]. Some techniques use the genetic algorithm, emphasizing the similarity of the procedure of 
the genetic algorithm to a traditional composition process. The genetic algorithm first generates tentative melodies and then explores better melodies repeatedly by partially changing those melodies. This process is very close to human composition process, in which human composers create a melody, play it, listen to it, and then correct it if necessary [12].

We have two challenges in using the genetic algorithm. The first is how to design a fitness function. The main advantage in the genetic algorithm relative to other techniques such as Makov models is the ability to use global features as well as local features such as $N$-gram probabilities. In fact, Ponce de León et al. [3] used global statistic descriptors such as the number of notes, the number of rests, average pitches, the largest pitch interval, etc. As a global feature, we introduce the similarity of a melody's entropy (how various note names appear) to those of existing melodies. By using this, we avoid excessively monotonous melodies such as C-D-C-D-C-D-...

The second is how to reduce the computation time. In general, the genetic algorithm is a technique that requires a computation cost. However, our system must generate melodies immediately because our aim is to support improvisation. We introduce two ideas to reduce the computation cost. One is to generate a melody individually for each measure to reduce the search space. The other is to generate initial chromosomes from existing melodies. A set of existing melodies are memorized as a prefix tree (called a melody tree). Initial chromosomes are generated by randomly proceeding in this prefix tree from the root. The initial chromosomes generated in this way are expected to be musically appropriate because they are generated from existing melodies, and the optimization of these chromosomes would quickly converge.

\section{System overview}

Figure 1 shows a screenshot of our system. Once the system is launched, a piano-roll interface is displayed on the screen. During the playback of an accompaniment (given by a MIDI file with a chord transcription), the user can draw a melodic outline on the piano-roll screen with a computer mouse or touch pad as an input device. The melody is created individually for each measure. Once the mouse cursor enters the region of measure $m$ and then moves out from that region (or the mouse button is released there), the creation of a melody for measure $m$ starts. After the melody is created, the most likely expression parameters (the onset, duration, and energy for each note) are estimated. Generation of melody and expression parameters in the current implementation takes approximately $0.5 \mathrm{~s}$ and $0.2 \mathrm{~s}$. Thus, users are required to draw their melodic outlines one bar in advance.

\subsection{Drawing melodic outline}

During playback, the user draws a melodic outline $\{y(t)\}$ on the piano-roll screen. Here, $y(t)$ represents the pitch of the outline (basically equal to a note number 
but it takes a continuous value) at time $t$. The time resolution is an eighth-note triplet in the current implementation.

\subsection{Determining rhythm of melody}

Melody generation for measure $m$ initiates when the mouse cursor moves out from measure $m$ in the piano roll (i.e. it moves to measure $m+1$ ) or the mouse button is released. First, the rhythm (i.e., the melody notes' durations) is determined. The key idea is to generate a note onset at time points of high-variability of $y(t)$. This is achieved through the following steps:

1. A set of note onset candidates, $\mathcal{R}$, is defined. Each element of $\mathcal{R}$ is a 12 dimensional binary vector, where 1 stands for an onset and 0 stands for a non-onset. For example, $(1,0,0,0,0,0,1,0,0,0,0,0)$ represents a sequence of two half notes.

2. We decide a tentative rhythm $R^{\prime}$ from $\{y(t)\}$. The $i$-th element of $R^{\prime}$, denoted by $R^{\prime}(i)$, is defined as follows:

$$
R^{\prime}(i)=\left\{\begin{array}{l}
1\left(\left|y\left(t_{m}+i\right)-y\left(t_{m}+i-1\right)\right|>\delta\right) \\
0 \text { (otherwise) }
\end{array}\right.
$$

where $t_{m}$ is the start time of $m$, and $\delta$ is a threshold.

3 . We search for the closest candidate to $R^{\prime}$, that is,

$$
\hat{R}=\underset{R_{k} \in \mathcal{R}}{\operatorname{argmin}}\left\|R_{k}-R^{\prime}\right\| .
$$

\subsection{Determining pitches}

For each of the 1-value elements in $\hat{R}$, the pitch (MIDI note number) is determined. Let $L$ be the number of 1 -value elements in $\hat{R}$. What should be determined here is $N=\left(n_{0}, \cdots, n_{L-1}\right)$, where $n_{i}$ is a note name (i.e., the remainder of the division of a MIDI note number by 12). To determine these pitches, we use a genetic algorithm $(\mathrm{GA})$, in which $N=\left(n_{0}, \cdots, n_{L-1}\right)$ is regarded as a chromosome. To achieve a quick melody creation, the optimization through GA is limited to $0.5 \mathrm{~s}$.

Initializing chromosomes Initial chromosomes are made from existing melodies. First, melodies taken from a melody corpus are memorized as a prefix tree (called a melody tree here). The basic concept of the melody tree is shown in Figure 2. Each node represents a note name, and each edge has the transition probability from the note name associated with the parent node to the note name associated with the child node. Those transition probabilities are calculated based on the melodies to be memorized. After all melodies are memorized, edges with very low transition probabilities are pruned. When the system generates initial chromosomes, it randomly proceeds in this tree from the root. 


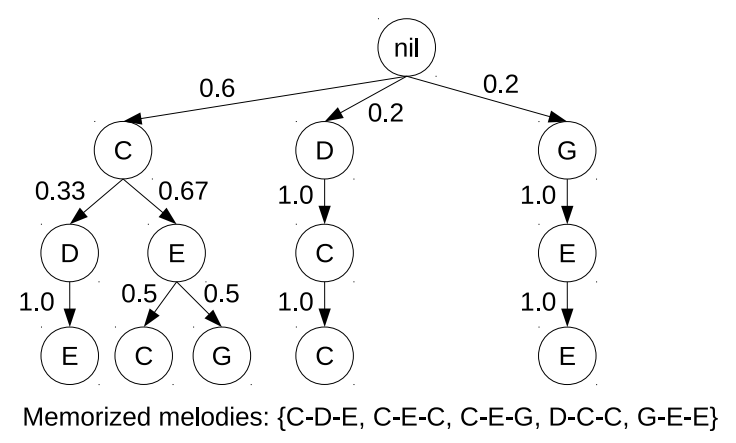

Fig. 2. Basic concept of a melody tree. Melodies are memorized in a prefix tree with transition probabilities.

Calculating fitness function The melodies to be generated should satisfy both similarity to the melodic outline drawn by the user and musical appropriateness. A typical approach for measuring the musical appropriateness of a melody is to calculate its $N$-gram probability based on the $N$-gram model trained with existing melodies. However, this approach has two problems. First, to use a large number for $N$, a tremendous number of data are required because the model size becomes very large (this is known as the data sparseness problem). Because the musical availability of each note depends on the chord, ideally the $N$-gram model should be switched according to the chord, but this could enhance the data sparseness problem. Second, the $N$-gram model considers only local characteristics in a melody. If only local characteristics are considered, monotonous melodies where the same local note transition is repeated, such as C-C-C-C-... or C-D-C-D-C-D-..., may be generated. For the first problem, we approximate the $N$-gram model using lower-order models. Specifically, we approximate a perchord trigram as a weighted sum of a bigram, a first-order derivative (so-called delta) bigram, and a per-chord unigram. For the second problem, we introduce the entropy of a melody as a global feature.

Thus, the fitness function $F(N)$ is defined as follows:

$$
F(N)=w_{0} \operatorname{sim}(N)+w_{1} \operatorname{seq}_{1}(N)+w_{2} \operatorname{seq}_{2}(N)+w_{3} \operatorname{harm}(N)+w_{4} \operatorname{ent}(N),
$$

where

$-\operatorname{sim}(N)$ : Similarity to outline

$$
\operatorname{sim}(N)=-\sum_{i=0}^{L-1}\left(n_{i}-y\left(t_{i}\right) \bmod 12\right)^{2},
$$

in which $t_{i}$ is the onset time of note $n_{i}$, and mod is the modulo operator. $-\operatorname{seq}_{1}(N)$ : Bigram probability

$$
\operatorname{seq}_{1}(N)=\sum_{i=1}^{L-1} \log P\left(n_{i} \mid n_{i-1}\right) .
$$


- $\operatorname{seq}_{2}(N):$ Delta bigram probability

$$
\operatorname{seq}_{2}(N)=\sum_{i=2}^{L-1} \log P\left(n_{i}-n_{i-1} \mid n_{i-1}-n_{i-2}\right) .
$$

- $\operatorname{harm}(N)$ : Per-chord unigram probability

$$
\operatorname{harm}(N)=\sum_{i=0}^{L-1} \log P\left(n_{i} \mid c_{i}, b_{i}\right),
$$

in which $c_{i}$ is the chord name at time $t_{i}$, and $b_{i}$ is the metrical position at $t_{i}$ $\left(b_{i} \in\{\right.$ head, on-beat, off-beat $\left.\}\right)$. We consider $b_{i}$ because the acceptability of out-of-scale notes depends on their metrical positions.

$-\operatorname{ent}(N)$ : Entropy

$$
\operatorname{ent}(N)=-\left(H(N)-H_{\text {mean }}-\varepsilon\right)^{2},
$$

in which $H(N)$ is the entropy of $\left\{n_{0}, \cdots, n_{L-1}\right\}$, and $H_{\text {mean }}$ is the averaged entropy calculated from a melody corpus. Above, $\varepsilon$ is usually zero, but setting this to more than zero will result in more complex melodies.

Above, $P\left(n_{i} \mid n_{i-1}\right), P\left(n_{i}-n_{i-1} \mid n_{i-1}-n_{i-2}\right), P\left(n_{i} \mid c_{i}, b_{i}\right)$, and $H_{\text {mean }}$ are learned from a corpus, while $w_{0}, \cdots, w_{4}$ and $\varepsilon$ are set manually.

\subsection{Estimating expression parameters}

The expression parameters, that is, the onset deviation, duration ratio, and energy (velocity) ratio for each note are estimated with Giraldo's method [7]. In this method, various features such as the pitch, duration, and Narmour structure description are extracted from each note, and the onset deviation, duration ratio, and energy ratio is estimated based on a machine learning model such as a $k$-nearest neighbor, a multi-layer perceptron, and a support vector machine. Here, we use the $k$-nearest neighbor estimator. The upper and lower bounds, if necessary, can be given to each parameter.

\section{Implementation}

We implemented this system on a touch-screen laptop PC. We used Groovy and CrestMuse Toolkit ${ }^{3}$ as a programming language and an implementation framework, respectively. For GA, we used the Apache Coomons Mathematics Library ${ }^{4}$. For feature extraction in estimating expression parameters, MIDI Toolbox ${ }^{5}$ for Matlab is used through JavaOctave ${ }^{6}$, i.e., a library binding Java and Octave.

\footnotetext{
${ }^{3}$ http://cmx.osdn.jp/

${ }^{4}$ http://commons.apache.org/proper/commons-math/

${ }^{5}$ https://github.com/miditoolbox/

${ }^{6}$ https://kenai.com/projects/javaoctave/pages/Home
} 
As a melody corpus, we used 53 melodies with the tonality of Blues taken from Weimar Jazz Database ${ }^{7}$. The total number of the measures and notes are 3,533 and 23,142 , respectively.

The default accompaniment is a 12-bar blues chord progression in the key of C, i.e., | C7 F7 C7 C7 $\quad$ F7 F7 C7 C7 G7 F7 C7 G7 |. This 12-bar chord progression is repeated four times. The accompaniment and repeat time can be changed; any accompaniment can be used if presents as a standard MIDI file with a separate chord transcription.

\section{$5 \quad$ Perceptual melody quality test}

We conducted a perceptual melody quality test based on a Turing-test-like approach. Specifically, we collected human performances and the system's performances and asked listeners to rate their quality. If the ratings for human performances and the system's performances have no significant differences, then the system can be considered to be capable of creating melodies that have equivalent quality to human performers.

\subsection{Procedure and participants}

Collection of improvisational melodies We asked participants to improvise with both our system and a normal MIDI keyboard. The procedure is as follows:

1. Recieve instructions on how to use the system.

2. Try improvisation with the system.

3. Practice improvisation with the system freely.

4. Improvise with the system again. This improvisation data will be used in a later listening test.

5. Try improvisation with the normal MIDI keyboard.

6. Practice improvisation with the normal MIDI keyboard freely.

7. Improvise with the normal MIDI keyboard again. This improvisation data will be used in the listening test.

The participants are the following six persons:

- $P_{1}$ : PostDoc researcher in sound/music computing, has a long experience in playing improvisation with the accordion,

- $P_{2}$ : plays the piano for about 15 years, composes musical pieces on a computer as a hobby, has not improvised,

- $P_{3}$ : plays the trumpet for 11 years, cannot improvise,

- $P_{4}$ : has no experience in music,

$-P_{5}$ : plays the Electone for 12 years, has composed only a little bit before, has not improvised,

- $P_{6}$ : plays the piano for about 15 years, plays improvisation as a professional player.

\footnotetext{
$\overline{7}$ http://jazzomat.hfm-weimar.de/dbformat/dboverview.html
} 

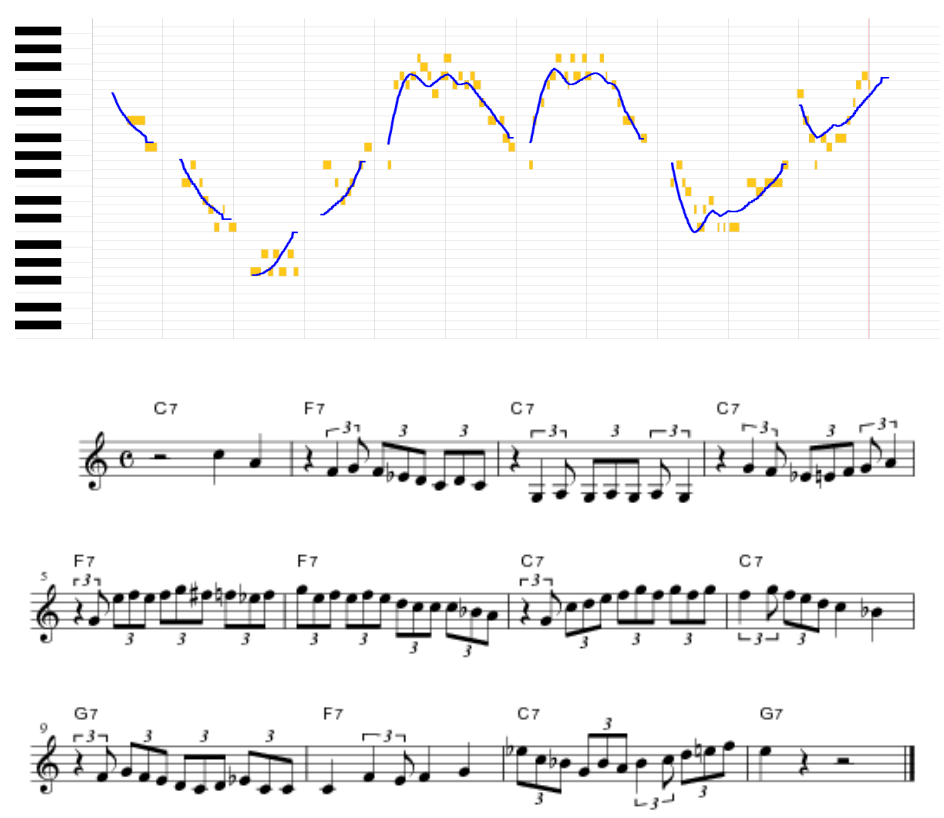

Fig. 3. The melodic outline and generated melody for 3rd loop in $s_{4}$.

Improvisation with the normal MIDI keyboard (Steps 5 to 7 ) was skipped in participants with no experience in playing an keyboard instrument $\left(P_{3}\right.$ and $\left.P_{4}\right)$. For advanced performers $\left(P_{1}\right.$ and $\left.P_{6}\right)$, improvisation with our system, Steps 1 to 4 , were skipped because they were not our target users.

Eventually, we collected the following performances:

- System's performances: $s_{1}$ (by $P_{2}$ ), $s_{2}$ (by $P_{3}$ ), $s_{3}$ (by $P_{4}$ ), and $s_{4}$ (by $P_{5}$ ),

- Human performances: $u_{1}$ (by $P_{1}$ ), $u_{2}$ (by $P_{2}$ ), $u_{3}$ (by $P_{5}$ ), and $u_{4}$ (by $P_{6}$ ).

Listening test We asked 12 researchers and students in music-related fields to evaluate each performance from the following criteria on a scale of 0 to 10:
1. Overall quality
2. Pitch appropriateness
5. Blues-likeness
3. Rhythmic appropriateness
6. Skillfulness
4. Melodic variation
7. Human-likeness

\subsection{Example of generated melodies}

The melodic outline and generated melody of the third loop in $s_{4}$ are shown in Figure 3. The generated melody is along the melodic outline and uses some blue notes $(\mathrm{E} b$ and $\mathrm{B} b)$. 

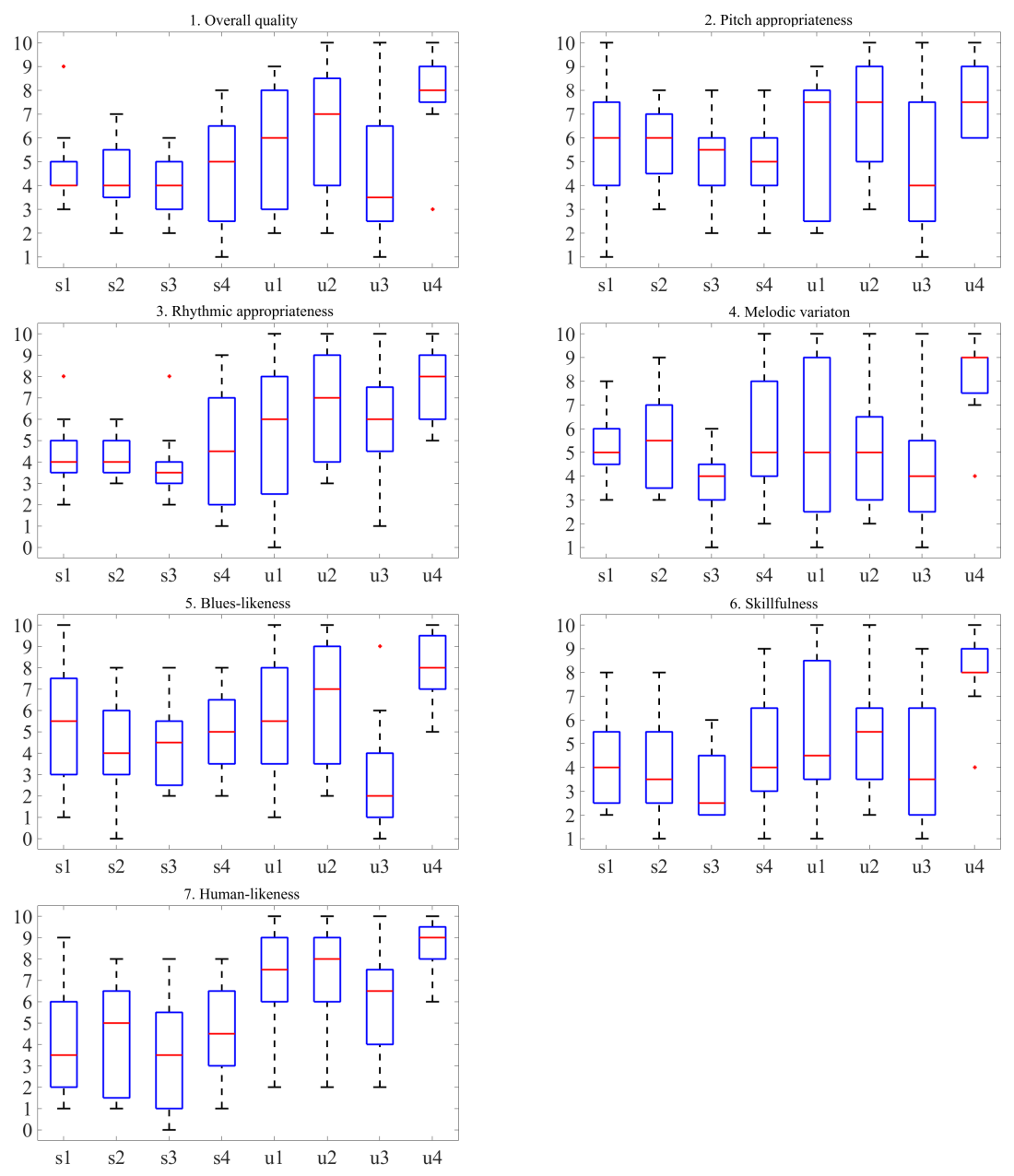

Fig. 4. Results of perceptual melody quality test

\subsection{Results}

The maximum value, 3rd quartile, median, 1st quartile, and minimum value of the ratings by the 12 listeners for each performance with respect to each criterion are shown in Figure 4. From these figures, we can see the following observations:

1. Overall quality

The performance $u_{4}$ has the highest median score. The performances that have the second and third highet median scores are $u_{2}$ and $u_{1}$, respectively. For system performances $\left(s_{1}\right.$ to $\left.s_{4}\right)$, the median scores are between 4 and 5 , which is higher than that for $u_{3}$.

2. Pitch appropriateness

The median scores for $u_{1}, u_{2}$, and $u_{4}$ are 7.5. Those for $s_{1}$ to $s_{4}$ are between 5 and 6 , which is higher than that for $u_{3}$. 
3. Rhythmic appropriateness

In general, human performances $\left(u_{1}\right.$ to $\left.u_{4}\right)$ have higher scores than the system's performances $\left(s_{1}\right.$ to $\left.s_{4}\right)$.

4. Melodic variation

The performance that has the highest median score is $u_{4}$. Other performances do not show clear differences in their scores.

5. Blues-likenesss

Also for this criterion, $u_{4}$ has the highest median value. The performance with the second highest median score is $u_{2}$. The system's performances $s_{1}$ to $s_{4}$ have median scores between 4 and 5.5 , which are almost equal to $u_{1}$ and definitely higher than that for $u_{3}$.

6. Skillfulness

The performance $u_{4}$ has the highest median score. The performance with the next highest median score is $u_{2}$. Other performances do not have clear differences in their median scores, between 2.5 and 4.5.

7. Human-likeness

The human performances $u_{1}$ to $u_{4}$ and the system's performances $s_{1}$ to $s_{4}$ have clear differences in their median scores. The former is between 6.5 and 9 while the latter is between 3.5 and 5 .

These results can be summarized as follows:

- The system's performances are better than a novice's performance, especially in pitch selection and blues-likeness.

- The system's performances have close quality to human performances except for advanced performers.

- The system's performances are inferior to a professional-level human performer.

- The system's performances have less human-likeness than human performances even a novice's performance.

\section{Conclusion}

Here, we proposed an improvisation support system called JamSketch, in which the user can enjoy improvisation just by drawing a melodic outline on the pianoroll display with the mouse or touch screen. Once the user draws a melodic outline, a melody is generated based on the genetic algorithm. Generated melodies are not as good as performances by advanced human players but are better than inexperienced players. From experiment participants, we obtained the following opinions:

- "I was able to play improvisation with less pressure than a normal MIDI keyboard."

- "I was able to try a wide pitch range, while I use only a familiar pitch range with a normal MIDI keyboard."

- "I was able to input my musical image intuitively." 
This feedback suggests that our system can provide non-musicians with a new way of music activity.

The main problem in the current melody generation method lies in the rhythmic aspect. Because the rhythm is determined by a simple rule, generated melodies often keep similar rhythms from beginning to end. In the future, we will improve the rhythm determination method using machine learning techniques, considering the dependency between the pitch and rhythm.

\section{References}

1. E. Amiot, T. Noll, M. Andretta, and C. Agon. Fourier oracles for computer-aided improvisation. In Proc. Int'l Computer Music Conf., 2006.

2. J. Buchholz, E. Lee, J. Klein, and J. Borchers. coJIVE: a system to support collaborative jazz improvisation. In Technical Report, Aachener Informatik-Berichte RWTH Aachen, Department of Computer Science, 2007.

3. P. J. P. de León, J. M. Inesta, J. Calvo-Zaragoza, and D. Rizo. Data-based melody generation through multi-objective evolutionary computation. J. Mathematics and Music, 10(2):173-192, 2016.

4. M. M. Farbood, E. Pasztor, and K. Jennings. Hyperscore: A graphical sketchpad for novice composers. IEEE Computer Graphics and Appl., 24(1):50-54, 2004.

5. S. Fels, K. Nishimoto, and K. Mase. MusiKalscope: A graphical musical instrument. IEEE Multimedia, 5(3):26-35, 1998.

6. J. Garcia, T. Tsandilas, C. Agon, and W. Mackay. Inksplorer: Exploring musical ideas on paper and computer. In Proc. Int'l Conf. on New Interfaces for Musical Expression, 2011.

7. S. Giraldo and R. Ramírez. A machine learning approach to ornamentation modeling and synthesis in jazz guitar. J. Mathematics and Music, 10(2):107-126, 2016.

8. K. Ishida, T. Kitahara, and M. Takeda. ism: Improvisation supporting system based on melody correction. In Proc. Int'l Conf. on New Interfaces for Musical Expression, pages 177-180, June 2004.

9. R. M. Keller. Welcome to impro-visor: Jazz improvisation advisor for the improviser. https://www.cs.hmc.edu/ ${ }^{\sim}$ keller/jazz/improvisor/.

10. M. Mandanici and S. Canazza. The "Hand Composer": Gesture-driven music composition machines. In Proc. 13th Conf. on Intelligent Autonomous Systems, pages 553-560, 2014.

11. H. Miyashita and K. Nishimoto. Theremoscore: A new-type musical score with temperature sensation. In Int'l Conf. New Interface for Musical Expression, 2004

12. G. Nierhaus. Algorithmic Composition. Springer, 2009.

13. F. Pachet. The Continuator: Musical interaction with style. In Proc. Int'l Computer Music Conf., 2002.

14. D. E. Parson. Chess-based composition and improvisation for non-musicians. In Proc. Int'l Conf. on New Interfaces for Musical Expression, 2009.

15. J.-B. Thiebaut, P. G. Healey, and N. B. Kinns. Drawing electroacoustic music. In Proc. Int'l Computer Music Conf., 2008.

16. Y. Tsuchiya and T. Kitahara. Melodic outline extraction method for non-notelevel melody editing. In Proc. Sound and Music Computing Conf., pages 762-767, 2013.

17. I. Xenakis. Music composition tracks. in Composers and the Computer, C. Roads (Eds.), 1985. 